\title{
Personalized computed tomography - Automated estimation of height and weight of a simulated digital twin using a 3D camera and artificial intelligence
}

\section{Personalisierte Computertomografie - automatisierte Abschätzung von Größe und Gewicht durch Simulation eines digitalen Zwillings mit einer 3D-Kamera und künstlicher Intelligenz}

Authors

Frederik Geissler ${ }^{1,2}$, Rafael Heiß², Markus Kopp², Marco Wiesmüller², Marc Saake ${ }^{1,2}$, Wolfgang Wuest ${ }^{1,2,3}$, Andreas Wimmer 4 , Veronika Prell ${ }^{4}$, Michael Uder ${ }^{1,2,3}$, Matthias Stefan May, 2, 3

\section{Affiliations}

1 Friedrich-Alexander-University Erlangen-Nürnberg (FAU), Erlangen, Germany

2 Department of Radiology, University Hospital Erlangen, Erlangen, Germany

3 Imaging Science Institute, Erlangen, Germany

4 Siemens Healthcare $\mathrm{GmbH}$, Forchheim, Germany

Key words

$\mathrm{CT}$, machine learning, digital twin, 3D camera, automatic data collection

received 05.05 .2020

accepted 26.08.2020

published online 03.11 .2020

Bibliography

Fortschr Röntgenstr 2021; 193: 437-445

DOI 10.1055/a-1253-8558

ISSN 1438-9029

(c) 2020. Thieme. All rights reserved.

Georg Thieme Verlag KG, Rüdigerstraße 14,

70469 Stuttgart, Germany

Correspondence

Frederik Geissler

Department of Radiology, University Hospital Erlangen,

Maximiliansplatz 3, 91054 Erlangen, Germany

Tel.: +49/9131/8545636

Frederik.Geissler@uk-erlangen.de

\section{ABSTRACT}

Purpose The aim of this study was to develop an algorithm for automated estimation of patient height and weight during computed tomography (CT) and to evaluate its accuracy in everyday clinical practice.

Materials and methods Depth images of 200 patients were recorded with a $3 \mathrm{D}$ camera mounted above the patient table of a CT scanner. Reference values were obtained using a calibrated scale and a measuring tape to train a machine learning algorithm that fits a patient avatar into the recorded patient surface data. The resulting algorithm was prospectively used on 101 patients in clinical practice and the results were compared to the reference values and to estimates by the patient himself, the radiographer and the radiologist. The body mass index was calculated from the collected values for each patient using the $\mathrm{WHO}$ formula. A tolerance level of $5 \mathrm{~kg}$ was defined in order to evaluate the impact on weight-dependent contrast agent dosage in abdominal CT.

Results Differences between values for height, weight and BMI were non-significant over all assessments $(p>0.83)$. The most accurate values for weight were obtained from the patient information $\left(R^{2}=0.99\right)$ followed by the automated estimation via 3D camera $\left(R^{2}=0.89\right)$. Estimates by medical staff were considerably less precise (radiologist: $R^{2}=0.78$, radiographer: $R^{2}=0.77$ ). A body-weight dependent dosage of contrast agent using the automated estimations matched the dosage using the reference measurements in $65 \%$ of the cases. The dosage based on the medical staff estimates would have matched in $49 \%$ of the cases.

Conclusion Automated estimation of height and weight using a digital twin model from 3D camera acquisitions provide a high precision for protocol design in computer tomography.

Key points:

- Machine learning can calculate patient-avatars from 3D camera acquisitions.

- Height and weight of the digital twins are comparable to real measurements of the patients.

- Estimations by medical staff are less precise.

- The values can be used for calculation of contrast agent dosage.

\section{Citation Format}

- Geissler F, Heiß R, Kopp M et al. Personalized computed tomography - Automated estimation of height and weight of a simulated digital twin using a 3D camera and artificial intelligence. Fortschr Röntgenstr 2021; 193: 437-445 


\section{ZUSAMMENFASSUNG}

Ziele Ziel der Studie war es, einen Algorithmus zur Abschätzung von Größe und Gewicht der Patienten vor einer Computertomografie (CT) zu entwickeln und dessen Genauigkeit im klinischen Alltag zu evaluieren.

Material und Methoden Mit einer über dem Patiententisch montierten 3D-Kamera wurden Tiefenbilder von 200 Patienten aufgenommen und zusammen mit den Referenzwerten einer geeichten Waage und eines Maßbandes zum Trainieren eines maschinellen Lernalgorithmus verwendet. Dies erfolgte über die Anpassung eines Patienten-Avatars an die aufgenommenen Oberflächeninformationen. Der so entstandene automatische Algorithmus wurde dann prospektiv im klinischen Alltag an 101 Patienten angewandt und die Ergebnisse mit den gemessenen Referenzwerten, den Patientenangaben sowie den Schätzwerten durch das technische und ärztliche Personal verglichen. Der Body-Mass-Index wurde aus den erhobenen Werten für jeden Patienten mit der WHO-Formel berechnet. Um die Auswirkungen auf die Kontrastmittel- menge bei gewichtsabhängiger Dosierung abschätzen zu können, wurde eine Toleranz von $5 \mathrm{~kg}$ definiert.

Ergebnisse Die Unterschiede zwischen den erhobenen Werten für Größe, Gewicht und BMI waren für alle Methoden nicht signifikant $(p>0,83)$. Die genauesten Werte für das Gewicht wurden aus der Patientenangabe $\left(R^{2}=0,99\right)$, gefolgt von der automatischen Erfassung über die 3D-Kamera $\left(R^{2}=0,89\right)$, erzielt. Abschätzungen durch das medizinische Personal waren deutlich ungenauer (Radiologe: $R^{2}=0,78$; MTRA: $\left.R^{2}=0,77\right)$. Eine gewichtsabhängige Kontrastmitteldosierung mit den Werten der automatischen Abschätzung wäre im Vergleich zur Dosierung anhand der Referenzmessungen in $65 \%$ der Fälle identisch gewesen. Analog wären bei einem Vorgehen basierend auf Schätzwerten des Personals $49 \%$ der Patienten identisch dosiert worden.

Schlussfolgerung Die automatisierte Abschätzung von Größe und Gewicht mit einer 3D-Kamera kann durch ein digitales Zwillingsmodell mit hoher Präzision für die Untersuchungsplanung in der Computertomografie verwendet werden.

\section{Introduction}

In Germany, computed tomography (CT) is the most frequently performed slice imaging technique, with growth of over $45 \%$ between 2007 and 2016 [1]. Many of the advantages for patient care described in the literature, e. g. reduction of tube voltage, spectral hardening using tin filters or dual energy, require a high degree of training of the examiners and a precise knowledge of the patient's physical characteristics [2-5]. The contrast medium dose can be individually adjusted as a function of tube voltage and body weight. George et al. showed that an individually-calculated quantity of contrast based on the patient's body weight ensures at least the same image quality as a standard dose protocol [6]. The interindividual variability of the measured image contrast is even lower compared to a standard protocol; the results are more comparable [7], while at the same time the amount of applied contrast was reduced on average. This results in a reduced risk to patients of contrast-induced acute renal failure, especially in cases of preexisting chronic renal insufficiency or when taking nephrotoxic drugs $[8,9]$. Compared to a standard protocol, a cost saving for the institution performing the procedure can be expected [10, 11]. The recommended procedure for determining the patient's weight is based on the patient's medical history or measurement using a scale. An estimate by the examiner was described as less precise [7]. In everyday clinical practice, this procedure is often limited, e.g. with unconscious, immobile or uncooperative patients. New 3D camera systems, which record surface and depth data using infrared technology offer a promising solution to this issue [12]. In newer computer tomographic systems, avatars are fitted as digital twins into the surface information of the positioned patients using machine learning. Saltybaeva et al. have already achieved significantly improved, automated patient positioning compared to manual positioning [13] which reduces radiation dose while improving image quality [14]. The aim of this study is to develop a 3D camera algorithm for the estimation of body height and weight and to evaluate it in clinical routine with respect to precision and contrast agent dosage. According to the null hypothesis, the estimated values provided by the digital twin, the patients' history data and estimates by the medical staff should differ significantly from the real measured values of height, body weight and BMI.

\section{Materials and Methods}

\section{Patients}

The study was coordinated with the local ethics committee and approved. After detailed information, all patients agreed in writing to participate. Over a period of three months, 321 randomlyselected patients aged 21 to 92 years with indication for computed tomography were enrolled in the study. Underage, non-compliant and immobile patients who could not be weighed were excluded. The examined body region was irrelevant to the preselection. All patients were examined using a third-generation dual source CT (Somatom Force, Siemens Healthcare GmbH, Forchheim, Germany).

\section{Size and weight}

After the disclosure, the weight and height of the patients were first visually estimated by the Medical-Technical Radiology Assistant (MTRA) and the radiologist, and the values were noted. Subsequently, the patient data were queried. As gold standard, the patients were weighed with a calibrated scale and measured with a wall-mounted tape measure (Seca Type 877 and Type 206, Seca $\mathrm{GmbH} \&$ Co KG, Hamburg, Germany). The average values of our cohort $(61.0 \pm 13.4$ years, $79.2 \pm 18.7 \mathrm{~kg}, 170.6 \pm 9.7 \mathrm{~cm}$, 


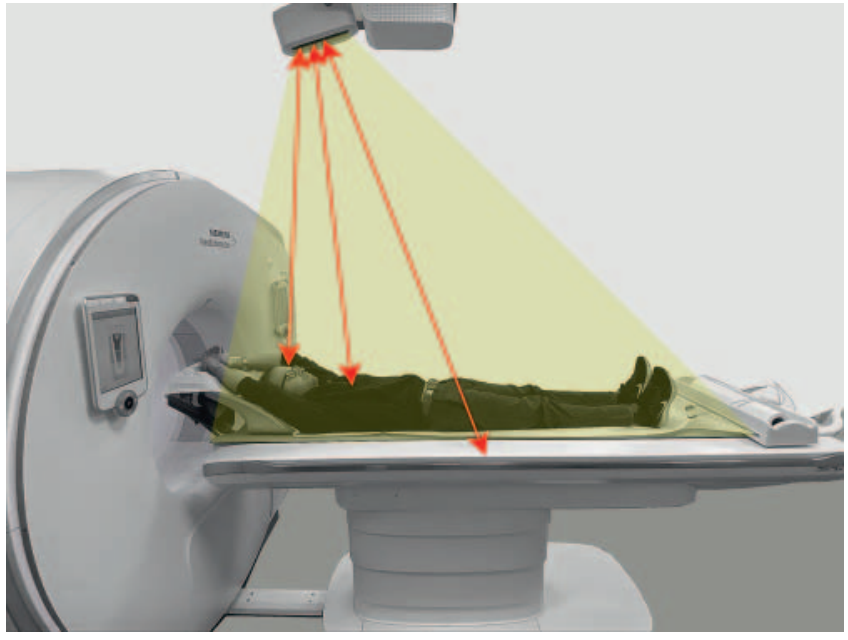

- Fig. 1 Operating principle of the Time-of-Flight (TOF) technique used by the 3D camera. Red double arrows = quickly and repeatedly emitted infrared laser pulses, reflected by patients body surface. Yellow $=$ camera field of view .

$27.1 \pm 5.4 \mathrm{~kg} / \mathrm{m}^{2}$ ) largely correspond to the figures of the Federal Statistical Office [15]. Prior to the weighing process, all loose objects and, if possible, footwear were removed. In view of the applicability and acceptance of the procedure in everyday clinical practice, complete undressing was deliberately avoided. To enable a systematic consideration of the clothing articles, it was noted whether street clothes $(n=303)$ or patient gowns $(n=18)$, shoes $(n=193)$ or no shoes $(n=128)$ were worn. To approximate the resulting measurement error, 20 different outfits or shoes of each category were weighed and their mean value calculated (street clothes $0.3 \mathrm{~kg}$, street shoes $0.5 \mathrm{~kg}$, patient gowns $0.2 \mathrm{~kg}$, slippers $0.3 \mathrm{~kg}$ ). The "corrected measured value" describes the difference between weight measurement and determined measuring error.

\section{D camera}

Prior to the start of the study, the combination of color and depth camera (Kinect 2.0, Microsoft Corp., Redmond Washington, USA) mounted above the patient table was calibrated. The depth information was collected using the time-of-flight (TOF) method, a measurement of time from the transmitted infrared pulse to the detection of the reflection (see $>$ Fig. 1). After completion of the individual positioning of the patients, depending on the planned examination and personal abilities, a reference image was taken by the examiner via a touch-pad interface permanently mounted on the gantry. In some cases, the patient was not completely captured in the manually triggered image, but had, for example, already partially entered the gantry. In such cases, a recording from a video sequence was used, which was simultaneously recorded by the camera system during the entire examination period and saved on an external storage system. In a total of 20 cases, it was not possible to obtain a complete image of the patient; accordingly the data set was subsequently excluded from the study.

Based on the first 100 patients, an existing algorithm for the detection of patients in combined color/depth data was expanded

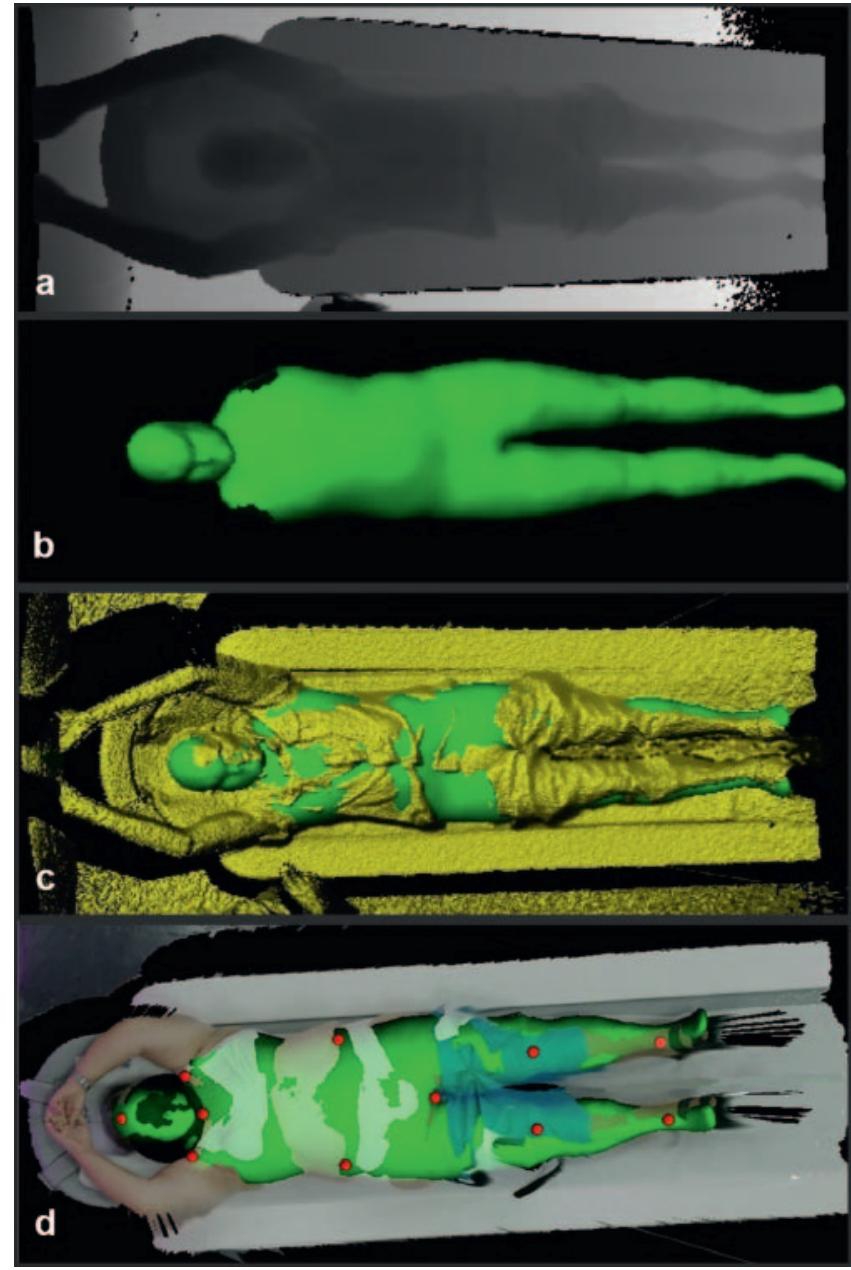

- Fig. 2 a-d Example of the algorithms method of operation when used on a new patient. a 3D-Pixelcloud (raw data), b Rendered avatar from the 3D-Pixelcloud, c Merging the avatar into the camera picture, $\mathbf{d}$ Automatic assignment of anatomical landmarks as defined in the training phase of the algorithm.

by the estimation of patient height and weight [16]. The essential steps are shown in $\mathbf{F i g . 2}$. Based on machine learning, the algorithm initially detects the patient's position and anatomical landmarks, including, e. g., the forehead, chin, shoulders, hips, crotch, knees and ankles (see $>$ Fig. 2d). Based on the landmarks, a virtual patient model, an "avatar", is then fitted into the depth data. This avatar is a statistical shape model based on a collection of 3D human scans and describes typical variations with respect to body height and proportions. The avatar is iteratively optimized, acquiring the position and body proportions found in the depth data (see $>$ Fig. 2c); deformations are limited by the statistical shape model to realistic body shapes. In this way, for example, broad items of clothing, blankets or headrests can be separated from the patient. The estimated body height corresponds directly to the length of the fitted avatar. Assuming an average density for human tissue, the weight can be estimated from the volume of the avatar. The avatar was broken down into regions such as head, torso, abdomen and legs in order to make the approach more flexible and allow for different weight distributions. Param- 
eters such as the volume and the dimensions of a "bounding box" of the sub-area are determined for each region. Using these parameters, a linear regressor was developed to establish the patient weight. Due to the large degrees of freedom and the difficulty of segmenting them accurately, the arms are not explicitly modeled, but the assumed proportional weight of the arms is later added to the estimation. The algorithm was optimized for 100 additional cases. Based on comparison with the anamnestic values and the estimates by the medical staff, the evaluation was then based on the last 101 unseen data sets.

\section{Contrast agent}

All examinations involving the abdomen (abdomen, thoracic abdomen, cervical-thoracic abdomen) were examined weight-adapted with intravenous contrast agent (lomeprol, Imeron 350, Bracco IMAGING Deutschland GmbH, Konstanz, Germany; dosage: $400 \mathrm{mg}$ (iodine) $/ \mathrm{kg}=1.14 \mathrm{ml} / \mathrm{kg}$ [6]) using a power injector (Accutron CT-D, Medtron, Saarbrücken, Germany). All other patients were examined with a predefined contrast dose (neck $80 \mathrm{ml}$, thorax $60 \mathrm{ml}$, neck-thorax $100 \mathrm{ml}$ ). The differential of the weightadapted contrast dose to common protocols without adjustment (abdomen/thorax abdomen: $100 \mathrm{ml}$, cervical/thoracic abdomen: $140 \mathrm{ml}$ ) was calculated retrospectively. To calculate the expense difference, a contrast agent cost of $0.08 € / \mathrm{ml}$ was used.

\section{Subgroups}

After a review of all data sets, subgroup analyses were carried out for the estimates using the digital twin in order to examine potential disruptive factors of the algorithm and the suitability of different patient groups for machine recording. The standard position was defined as patient positioning with arms above the head, legs without knee wedge and pants not pulled down. Separately, subgroups with knee wedge, pants pulled down to the back of the knee and arms resting on the torso were considered. In addition, weight-related subgroups were analyzed. These were defined as normal and slightly overweight patients (Body Mass Index, BMI $\left.18-30 \mathrm{~kg} / \mathrm{m}^{2}\right)$, obese patients $(\mathrm{BMI}>30)$ and patients with a weight between the $1 \mathrm{st}$ and $3 \mathrm{rd}$ quartile of the training cohort (65-90 kg).

\section{Statistical Analysis}

Statistical analysis was performed with SPSS Version 24 (IBM Corp., Armonk, New York, USA) and Excel 2017 (Microsoft Corp., Redmond, Washington, USA). The deviations of the estimates $\left(E_{x}\right)$ from the gold standard $(G)$ are expressed as mean absolute error (MAE) and range of difference (R) as follows:

$M A E=\frac{1}{101} \sum_{i=1}^{101}\left|E_{x}-G\right|$

$R=\min \left\{\left(E_{x 1}-G_{1}\right) ; \ldots ;\left(E_{x 101}-G_{101}\right)\right\} ; \max \left\{\left(E_{x 1}-G_{1}\right) ; \ldots ;\left(E_{x 101}-G_{101}\right)\right\}$

A Kolmogorov-Smirnov test confirmed the normal distribution of the measurement results (significance level $\alpha<0.05$ ). The differences between the training and application cohorts were analyzed with unpaired t-tests. Variances of the individual methods of estimation from the corrected measurement results were analyzed using single factor analysis of variance (ANOVA).

\section{Results}

\section{Height and weight}

Weight, height and BMI in the training cohort $(79.9 \pm 19.5 \mathrm{~kg}$; $170.5 \pm 9.8 \mathrm{~cm} ; 27.3 \pm 5.5 \mathrm{~kg} / \mathrm{m}^{2}$ ) were not significantly different from the application cohort $(77.7 \pm 16.7 \mathrm{~kg} ; 170.7 \pm 9.7 \mathrm{~cm}$; $26.6 \pm 5.2 \mathrm{~kg} / \mathrm{m}^{2}$; all $\mathrm{p} \geq 0.126$ ). The corrected measured values of the weight were on average $0.6 \mathrm{~kg}$ below the documented values. Deviations of the mean values of the measuring methods were very small and not statistically significant, with maximum $2.0 \mathrm{~kg}$ body weight $(-2.6 \%, \mathrm{p}=0.900)$, maximum $0.8 \mathrm{~cm}$ body height $(-0.5 \%, p=0.927)$ and maximum $0.8 \mathrm{~kg} / \mathrm{m} 2(-3.0 \%, \mathrm{p}=0.830)$. Camera and patient underestimated the weight in the majority of cases (68\%). MTRA and radiologist, on the other hand, underestimated and overestimated in roughly equal proportions $(\sim 50 \%$, see $>$ Table 1$)$. Only one patient and two radiologist estimates exactly matched the corrected measurements

The patient shows the lowest MAE and the smallest range in all estimates. For the parameter weight, the patient is on average $2.9 \mathrm{~kg}$ closer to the corrected measurement value than the camera and $4.7 \mathrm{~kg}$ closer than radiologist and MTRA. The MAE of the camera estimation is below, the MAE of radiologist and MTRA above $5 \mathrm{~kg}$ estimation deviation. In terms of size and calculated BMI, all estimating groups show a comparable tendency (see - Table 2).

\section{Subgroup analyses}

In standard position, the mean values of weight $(-2.0 \%)$ and BMI $(-1.9 \%)$ with the digital twin were lower than the measured gold standard and almost equal to the height $(-0.0 \%$, see $>$ Table 3$)$. The absolute mean measurement error was lower than in the overall cohort (see $>$ Table 2). Four patients were underestimated by more than $5 \mathrm{~kg}$ and 2 patients were overestimated (23\% in total).

When positioned with a knee wedge, the mean values of weight (+5.1\%), height (+ $0.7 \%)$ and $\mathrm{BMI}(+3.5 \%)$ were higher. The absolute mean measurement error was higher than in the overall cohort ( $>$ Fig. 3 ). One patient was underestimated by more than $5 \mathrm{~kg}$ and 4 patients were overestimated (46\% in total).

When the pants were pulled down to knee level, the mean values of weight $(-2.6 \%)$, height $(-0.6 \%)$ and $\mathrm{BMI}(-1.8 \%)$ were below the reference. The absolute mean measurement error was barely higher than in the overall cohort. Four patients were underestimated by more than $5 \mathrm{~kg}$ and 7 patients were overestimated ( $40 \%$ in total).

When the arms were placed on the body, the mean values of weight $(-4.6 \%)$, height $(-0.5 \%)$ and $\mathrm{BMI}(-3.5 \%)$ were also lower. The absolute mean measurement error of weight and BMI was lower, while the mean measurement error of height was significantly higher than in the overall cohort. Two patients were underestimated by more than $5 \mathrm{~kg}$ and no patient overestimated ( $22 \%$ in total). 
- Table 1 Mean values and standard deviations of patient parameters depending on the estimation method with frequency of over- and underestimation of weight. No correction was needed for height.

\begin{tabular}{|c|c|c|c|c|c|c|}
\hline & amount & $\begin{array}{l}\text { average weight } \\
{[\mathrm{kg}]}\end{array}$ & $\begin{array}{l}\text { average height } \\
{[\mathrm{cm}]}\end{array}$ & $\begin{array}{l}\text { average BMI } \\
{\left[\mathrm{kg} / \mathrm{m}^{2}\right]}\end{array}$ & $\begin{array}{l}\text { frequency of } \\
\text { underestimation } \\
\text { (weight) }\end{array}$ & $\begin{array}{l}\text { frequency of } \\
\text { overestimation } \\
\text { (weight) }\end{array}$ \\
\hline measurement & 101 & $78.3 \pm 16.8$ & $170.7 \pm 9.8$ & 26.8 & - & - \\
\hline $\begin{array}{l}\text { corrected } \\
\text { measurement }\end{array}$ & 101 & $77.7 \pm 16.8$ & - & 26.6 & - & - \\
\hline camera & 101 & $75.7 \pm 15.2$ & $170.4 \pm 8.7$ & 26.0 & 68 & 33 \\
\hline patient & 101 & $77.1 \pm 16.3$ & $171.5 \pm 9.6$ & 26.1 & 68 & 32 \\
\hline radiologist & 101 & $76.6 \pm 14.4$ & $171.2 \pm 9.5$ & 26.1 & 47 & 52 \\
\hline radiographer & 101 & $76.1 \pm 14.7$ & $171.3 \pm 10.1$ & 25.8 & 54 & 47 \\
\hline
\end{tabular}

- Table 2 Mean absolute error (MAE) with standard deviation and range (R) for corrected weight, height and BMI depending on estimation method and - subgroup.

\begin{tabular}{|c|c|c|c|c|c|c|c|}
\hline & \multirow[t]{2}{*}{ amount } & \multicolumn{2}{|c|}{ weight [kg] } & \multicolumn{2}{|c|}{ height [cm] } & \multicolumn{2}{|c|}{ BMI $\left[\mathrm{kg} / \mathrm{m}^{2}\right]$} \\
\hline & & MAE & $\mathbf{R}$ & MAE & $\mathbf{R}$ & MAE & $\mathbf{R}$ \\
\hline camera & 101 & $4.4 \pm 3.9$ & $-16.1 ;+13.8$ & $2.5 \pm 1.9$ & $-6.9 ;+8.8$ & $1.6 \pm 1.3$ & $-5.1 ;+6.0$ \\
\hline - standard position & 26 & $3.3 \pm 2.7$ & $-11.5 ;+5.6$ & $2.2 \pm 1.8$ & $-6.4 ;+5.1$ & $1.3 \pm 0.9$ & $-3.6 ;+2.5$ \\
\hline - knee wedge & 11 & $5.2 \pm 4.3$ & $-6.0 ;+13.8$ & $2.6 \pm 1.5$ & $-3.0 ;+5.2$ & $2.1 \pm 1.8$ & $-3.7 ;+6.0$ \\
\hline - pulled down trousers & 57 & $5.2 \pm 4.5$ & $-16.1 ;+13.8$ & $2.6 \pm 1.9$ & $-6.8 ;+8.8$ & $1.8 \pm 1.5$ & $-5.1 ;+6.0$ \\
\hline - arms over body & 9 & $4.0 \pm 4.8$ & $-16.1 ;+0.8$ & $3.0 \pm 1.3$ & $-5.5 ;+3.4$ & $1.3 \pm 1.0$ & $-3.5 ;+0.9$ \\
\hline - BMI $18-30$ & 79 & $4.2 \pm 3.8$ & $-16.1 ;+13.8$ & $2.6 \pm 2.0$ & $-6.9 ;+8.8$ & $1.4 \pm 1.2$ & $-4.2 ;+6.0$ \\
\hline$-\mathrm{BMI}>30$ & 20 & $5.2 \pm 4.3$ & $-15.6 ;+7.3$ & $2.4 \pm 1.6$ & $-5.1 ;+5.2$ & $2.1 \pm 1.4$ & $-5.1 ;+1.8$ \\
\hline - weight: $65-90 \mathrm{~kg}$ & 50 & $3.4 \pm 3.3$ & $-13.0 ;+5.6$ & $2.3 \pm 1.8$ & $-6.9 ;+8.8$ & $1.3 \pm 1.0$ & $-4.2 ;+2.5$ \\
\hline patient & 101 & $1.5 \pm 1.3$ & $-6.7 ;+4.9$ & $1.8 \pm 1.5$ & $-4.0 ;+7.5$ & $0.8 \pm 0.7$ & $-3.2 ;+1.5$ \\
\hline radiologist & 101 & $6.2 \pm 4.9$ & $-22.9 ;+19.6$ & $2.3 \pm 1.7$ & $-7.0 ;+6.0$ & $2.1 \pm 1.7$ & $-8.4 ;+5.3$ \\
\hline radiographer & 101 & $6.2 \pm 5.9$ & $-35.5 ;+18.2$ & $3.2 \pm 2.6$ & $-10.5 ;+11.2$ & $2.2 \pm 2.1$ & $-13.3 ;+4.3$ \\
\hline
\end{tabular}

Normal weight or slightly overweight patients had mean values of weight $(-2.6 \%)$, height $(-0.2 \%)$ and BMI $(-1.7 \%)$ below the measured gold standard. The absolute mean measurement error was comparable to the overall cohort. Seventeen patients were underestimated by more than $5 \mathrm{~kg}$ and 6 patients were overestimated ( $29 \%$ in total).

Obese patients had mean values of weight (-3.9\%), height $(-0.1 \%)$ and BMI $(-4.7 \%)$ somewhat below the reference value. The absolute mean measurement error of weight and BMI was higher than the overall cohort, while the mean measurement error of height was comparable, however. Eight patients were underestimated by more than $5 \mathrm{~kg}$ and 2 patients were overestimated ( $50 \%$ in total).

Between the 1 st and 3 rd weight quartiles of the reference cohort, the mean values of weight $(-3.1 \%)$, height $(-0.2 \%)$ and BMI $(-2.9 \%)$ with the digital twin were lower than the gold standard. The absolute mean measurement error for all three parameters was lower than in the overall cohort. Eleven patients were underestimated by more than $5 \mathrm{~kg}$ and 1 patient was overestimated ( $24 \%$ in total).

\section{Correlation}

The highest correlation for the parameter weight was achieved by the patient estimate $\left(R^{2}=0.988\right)$, followed by the camera estimate $\left(R^{2}=0.891\right)$. Radiologists $\left(R^{2}=0.781\right)$ and MTRAs $\left(R^{2}=0.767\right)$ achieved comparably low correlation values $\left(\triangleright\right.$ Fig. 4). With respect to height, the patient $\left(R^{2}=0.948\right)$, camera $\left(R^{2}=0.900\right)$ and radiologist $\left(R^{2}=0.915\right)$ estimates correlate well, while the MTRA estimate was significantly lower $\left(R^{2}=0.832\right)$. The calculated BMI correlated well with patient data $\left(R^{2}=0.965\right)$ and camera $\left(R^{2}=0.857\right)$; radiologists $\left(R^{2}=0.735\right)$ and MTRA $\left(R^{2}=0.678\right)$ were lower. 
- Table 3 Comparison of measured mean values and standard deviations for patient parameters and camera calculations sorted by $\leadsto$ subgroup.

\begin{tabular}{|c|c|c|c|c|c|}
\hline & amount & method & $\begin{array}{l}\text { average weight } \\
\text { [kg] }\end{array}$ & $\begin{array}{l}\text { average height } \\
{[\mathrm{cm}]}\end{array}$ & $\begin{array}{l}\text { Average BMI } \\
{\left[\mathrm{kg} / \mathrm{m}^{2}\right]}\end{array}$ \\
\hline \multirow[t]{2}{*}{ - standard position } & \multirow[t]{2}{*}{26} & measurement & $75.0 \pm 15.8$ & $168.9 \pm 8.3$ & $26.2 \pm 4.9$ \\
\hline & & camera & $73.5 \pm 13.4$ & $168.9 \pm 7.8$ & $25.7 \pm 4.0$ \\
\hline \multirow[t]{2}{*}{ - knee wedge } & \multirow[t]{2}{*}{11} & measurement & $81.0 \pm 21.7$ & $167.4 \pm 9.9$ & $28.9 \pm 7.6$ \\
\hline & & camera & $85.1 \pm 18.5$ & $168.5 \pm 10.0$ & $29.9 \pm 5.9$ \\
\hline \multirow[t]{2}{*}{ - pulled down trousers } & \multirow[t]{2}{*}{57} & measurement & $80.9 \pm 17.0$ & $172.7 \pm 9.7$ & $27.1 \pm 5.5$ \\
\hline & & camera & $78.7 \pm 15.5$ & $172.0 \pm 8.7$ & $26.6 \pm 4.9$ \\
\hline \multirow[t]{2}{*}{ - arms over body } & \multirow[t]{2}{*}{9} & measurement & $81.8 \pm 11.4$ & $170.1 \pm 8.1$ & $28.3 \pm 3.6$ \\
\hline & & camera & $78.0 \pm 10.1$ & $169.3 \pm 7.1$ & $27.3 \pm 3.7$ \\
\hline \multirow[t]{2}{*}{ - BMI 18-30 } & \multirow[t]{2}{*}{79} & measurement & $73.4 \pm 13.3$ & $171.4 \pm 9.7$ & $24.9 \pm 3.2$ \\
\hline & & camera & $71.5 \pm 11.9$ & $170.7 \pm 8.4$ & $24.4 \pm 2.9$ \\
\hline \multirow[t]{2}{*}{$-\mathrm{BMI}>30$} & \multirow[t]{2}{*}{20} & measurement & $98.1 \pm 10.2$ & $169.2 \pm 9.4$ & $34.3 \pm 3.4$ \\
\hline & & camera & $94.3 \pm 10.6$ & $169.0 \pm 9.5$ & $32.7 \pm 3.4$ \\
\hline \multirow[t]{2}{*}{ - weight: $65-90 \mathrm{~kg}$} & \multirow[t]{2}{*}{50} & measurement & $76.9 \pm 6.6$ & $171.9 \pm 7.2$ & $26.1 \pm 2.6$ \\
\hline & & camera & $74.4 \pm 7.2$ & $171.5 \pm 6.4$ & $25.3 \pm 2.6$ \\
\hline
\end{tabular}

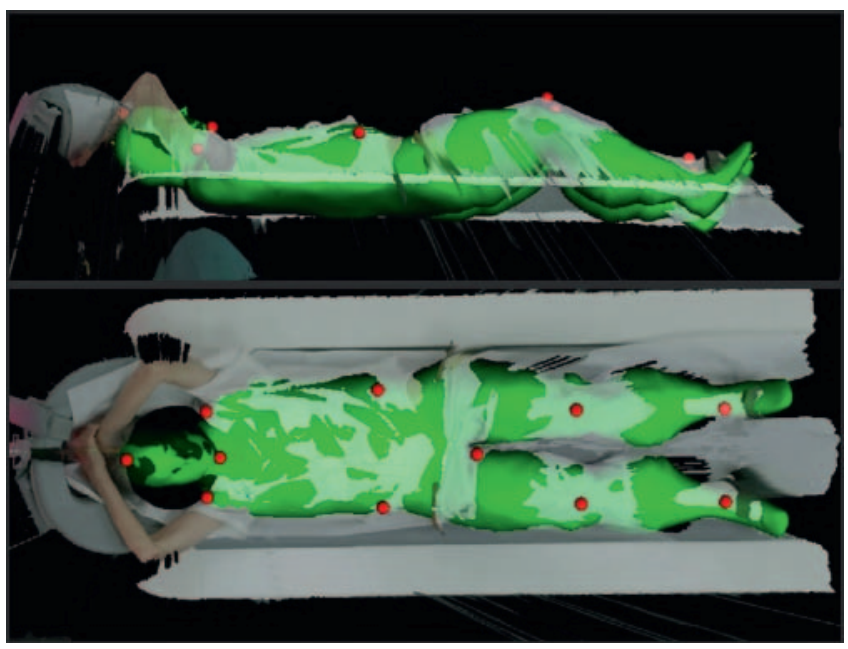

- Fig. 3 Influence of the knee wedge on the patient avatar: the thighs and lower legs are extrapolated too strongly, the patient thereby miscalculated as too heavy.

\section{Contrast agent}

With a threshold value of $5 \mathrm{~kg}$, analogous to the conversion table of Perrin et al., $35 \%$ of the patients would have been divided into a different dose group using the values of the camera algorithm [11]. In the majority of cases a dosage one step lower is chosen (71\%). The misjudgment rate of radiologists and MTRAs was significantly higher at $51 \%$ each. The majority of patients were underestimated by radiologists and MTRAs (63\% and 57\%, respectively). The patients underestimated themselves in only $2 \%$ of cases to an extent relevant for the contrast dosage ( $\vee$ Fig. 5).
Intravenous contrast was used to examine the abdomen in 87 of 101 patients. Compared to a standard protocol, weightadapted contrast administration saved an average of $10.3 \mathrm{ml}$ of contrast agent per patient and thus $0.82 €$ in material costs (see - Table 4).

\section{Discussion}

Our study shows no significant difference between the measured and estimated values for the parameters height, body weight and $\mathrm{BMI}$, regardless of the estimation method. All estimation groups tend to underestimate with a threshold value of $5 \mathrm{~kg}$. The camera algorithm calculates $25 \%$ of patients too light and $10 \%$ too heavy, thus achieving a $33 \%$ improvement over the estimate by radiologists and MTRAs. Patients with normal weight or slightly overweight (-6\% contrast-relevant misjudgments), in the interquartile range of the training collective $(-11 \%)$ and patients in a standardized position $(-12 \%)$ were significantly better assessed by the camera. Arms resting on the body were well compensated by the algorithm $(-13 \%)$. The false estimate rate was higher for obese patients $(+15 \%)$, those with a positioning knee wedge $(+11 \%)$ and for patients with their pants pulled down (+5\%). The majority of patients with knee wedge was the only subgroup calculated too heavily, because the algorithm extrapolates the resting leg parts too strongly.

With a total of only $2 \%$ relevant underestimated cases, the targeted patient history is the most accurate method for determining body weight. However, the mean estimation error of our patients $(1.5 \mathrm{~kg})$ was significantly lower than in the study by Benbow et al. $(2.8 \mathrm{~kg}$ ) [7]. In order to keep the burden of the examination as low as possible in the event a measurement or 


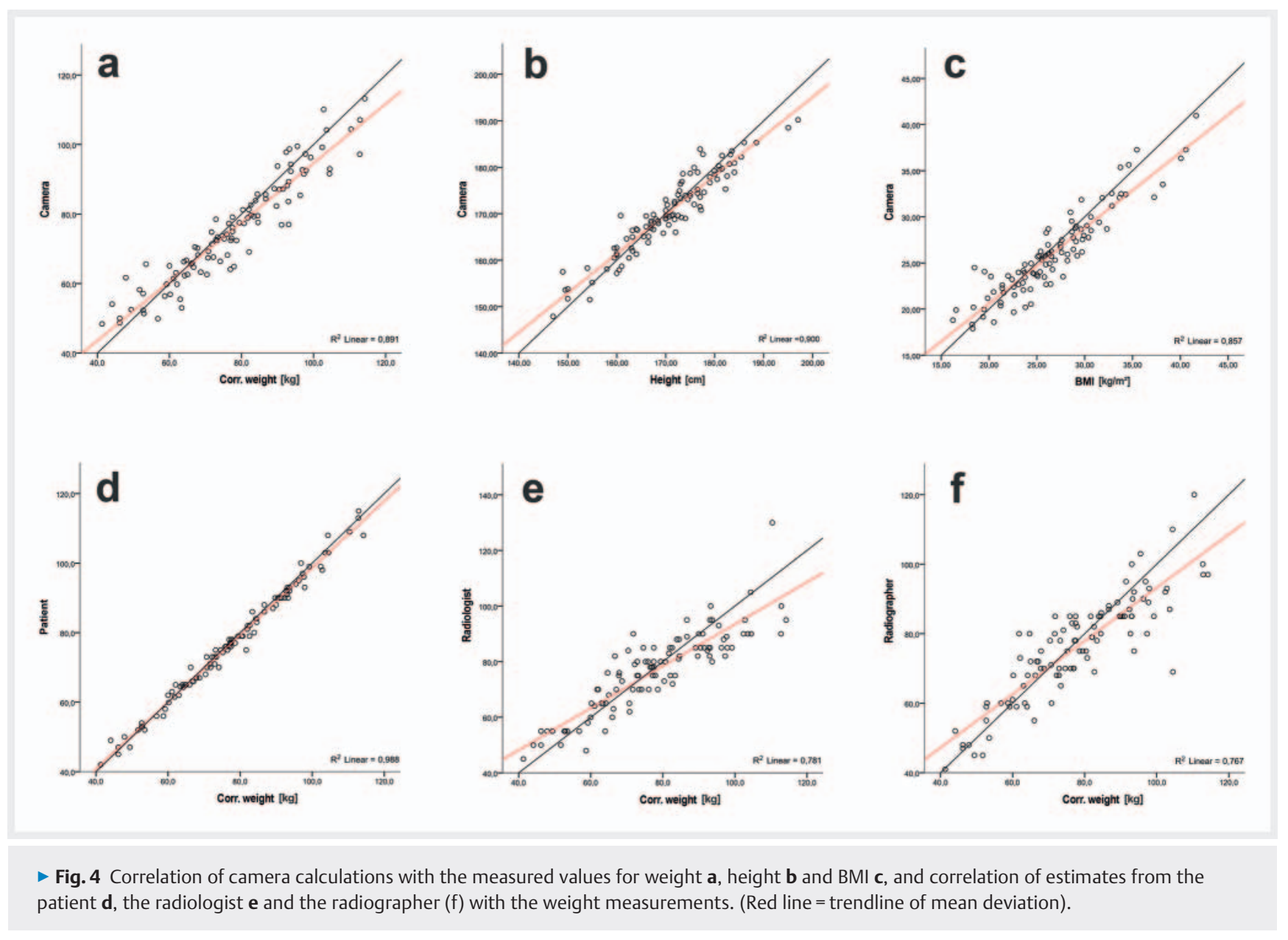

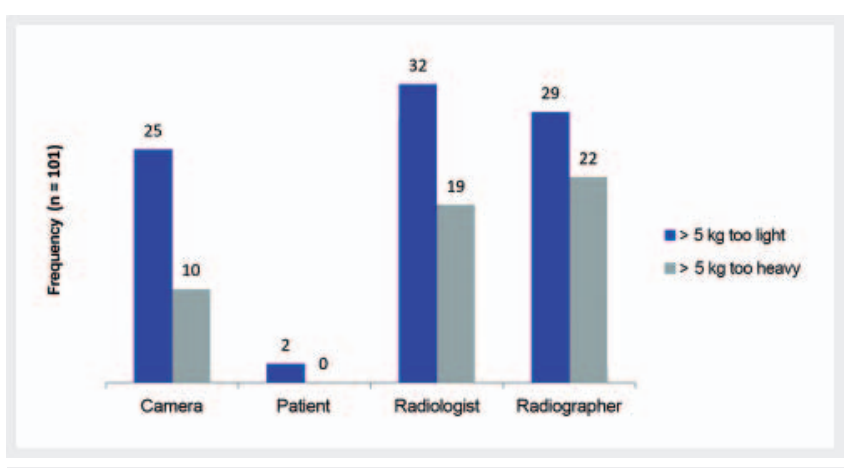

- Fig. 5 Frequency of relevant weight deviations $>|5 \mathrm{~kg}|$ of individual estimation groups.

anamnesis is not possible, e. g. in intensive care and trauma patients, the camera algorithm is better suited for assessment than the medical staff. The cost reduction assumed in the literature by several authors by the weight-adapted administration of contrast can be confirmed and precisely stated $(-7,995 €)$, when scaled for our institute with approx. 9750 contrast medium-supported abdominal examinations per year $[10,11]$.

An approach favored by Kondo et al. to calculate the ideal CT contrast agent dosage by determining the fat-free body mass using the body surface is currently not implemented in clinical routine due to the increased effort involved [17]. Automated acquisition could facilitate implementation and transfer the digitally available values via injector coupling without the need for additional interaction. This could also improve the workflow at high patient throughput, which is often seen as problematic, leaving more time for patient care $[7,11,18,19]$. In the future, the standardized values could also be used in combination with the DICOM image data set for the automatic calculation of surfacenormalized organ volumes. Standardized volume data, such as the expected residual volume of the liver, could make a decisive contribution to therapy in the planning phase, e. g. before oncological liver surgery, selective radioembolization or liver transplants [20-23]. Automatic implementation of the collected parameters in a digital patient file could also ensure rapid availability of relevant data, such as during follow-up examinations in other institutions or when determining the dose of medication in emergency situations without the possibility of patient history or measurement, for example before lysis therapy for stroke patients [12]. In the context of quality assurance, inclusion of this data supports investigation of causes for exceeding the $\mathrm{CT}$ dose reference values and to document justifiable reasons for this. For example, in the "Guidelines for the Handling of Diagnostic Reference Values in X-ray Diagnostics", the Federal Office for Radiation Protection explicitly recommends recording “[...] further data (e.g. the 
- Table 4 Contrast agent dosage and cost calculations with and without weight adaptation stratified into regions of investigation.

\begin{tabular}{|l|l|l|l|l|}
\hline & NTA & TA & A & total \\
\hline patients & 33 & 45 & 9 & 87 \\
\hline CA-Dosage not adapted & $4,620 \pm 0 \mathrm{ml}$ & $4,500 \pm 0 \mathrm{ml}$ & $900 \pm 0 \mathrm{ml}$ & $10,020 \pm 19.4 \mathrm{ml}$ \\
\hline CA-Dosage weight adapted & $4,168 \pm 19.5 \mathrm{ml}$ & $4,101 \pm 19.1 \mathrm{ml}$ & $859 \pm 15.6 \mathrm{ml}$ & $9,128 \pm 25.3 \mathrm{ml}$ \\
\hline CA-Dosage reduction & $452 \pm 19.5 \mathrm{ml}$ & $399 \pm 19.1 \mathrm{ml}$ & $41 \pm 15.6 \mathrm{ml}$ & $892 \pm 19.2 \mathrm{ml}$ \\
\hline cost reduction & $36.16 €$ & $31.92 €$ & $3.28 €$ & $71.36 €$ \\
\hline A - abdomen; TA - thorax-abdomen; NTA - neck-thorax-abdomen, CA - Contrast agent. & & \\
\hline
\end{tabular}

body diameter or the body weight of patients) [...]" for the evaluation of reasons when dose limits are exceeded [24].

Our study has limitations. Relatively few data sets $(n=200)$ were available for the development and training of the algorithm for a machine learning procedure. To minimize this problem, the algorithm was optimized in the training phase by cross-validation. The greater inaccuracy of the algorithm in heavily overweight and very slim patients is due to the small number of comparison patients in these subgroups. Likewise, there are no training data available for childlike body proportions. Because of the limited possibility to collect reference values, immobile patients could not be included for the development of the algorithm. However, due to standardized recording in a supine examination position, the algorithm can be applied to these patients regardless of the degree of immobilization. Further inaccuracies are due to incomplete recording of the arms and the general correction for garments. In a detailed evaluation of the greatest outliers, additional potential sources of error included a pointed foot position, black clothing and therefore an increased noise in the surface data as well as asymmetric simulation of the legs. With an increasing number of training data sets, however, it can be expected that the precision of the algorithm will also increase for the various subgroups. The obvious alternative of automated measurement using an electronic scale integrated in the examination table would be technically extremely complex, susceptible to damage and disproportionately expensive due to the high mechanical demands. Unlike surface detection using infrared technology, a scale cannot measure height and cannot differentiate between patients and other objects, e. g. a monitor. A further technical limitation results from the central positioning of the camera above the patient, which makes it harder to recognize body parts that depend on the side of the examination table and can therefore incorrectly calculate the avatar. One solution could be a second camera at the side of the table, making it possible to better identify positioning variants and aids such as a knee wedge.

\section{Conclusions}

Based on machine learning, images from a 3D camera can be used to model a digital avatar into the patient interface, which can then be used to accurately estimate patient height and weight. This automatic assessment provides better results than staff estimates especially in situations without reliable patient data. In the future, individual contrast adjustments could be implemented automatically and enable standardized image quality with minimal patient load even in critical situations. In addition, economic optimization of CT with time savings and cost reductions also appears possible. Digitally-structured values are directly available for further use and make it possible to open up new areas of application, for example in therapy planning.

\section{CLINICAL RELEVANCE OF THE STUDY}

- Compared to medical personnel, automated estimation of patient body constitution can provide more reliable values for individual examination adaptation, such as weightadapted contrast dosage in CT.

- Especially patients without a personal medical history, such as intensive care and trauma patients, can be spared unnecessary stress.

- Especially with a high workload, valuable working time could be saved, costs reduced and potential sources of error reduced.

\section{Conflict of Interest}

Die Autoren erklären, dass sie innerhalb der vergangenen 3 Jahre Forschungsunterstützung von Siemens Healthcare $\mathrm{GmbH}$ und Vortragshonorare von Siemens Healthcare GmbH erhalten haben.

\section{Acknowledgements}

Frederik Geissler performed the present work in partial fulfillment of the requirements for obtaining the degree "Dr. med.".

\section{References}

[1] Bundesamt für Strahlenschutz. Röntgendiagnostik: Häufigkeit und Strahlenexposition. https://www.bfs.de/DE/themen/ion/anwendung-medizin/ diagnostik/roentgen/haeufigkeit-exposition.html 
[2] Petritsch B, Kosmala A, Gassenmaier T et al. Diagnostik der akuten Lungenarterienembolie: Vergleich von Single-Source CT und Dritt-Generation Dual-Source CT unter Einsatz eines Dual-Energy Protokolls - Bildqualität und Strahlenexposition. Fortschr Röntgenstr 2017. doi:10.1055/s-0043-103089

[3] May MS, Eller A, Stahl C et al. Dose reduction in computed tomography of the chest: image quality of iterative reconstructions at a $50 \%$ radiation dose compared to filtered back projection at a $100 \%$ radiation dose. Fortschr Röntgenstr 2014. doi:10.1055/s-0033-1356254

[4] Do TD, Sutter R, Skornitzke S et al. CT- und MRT-Bildgebung bei orthopädischen Implantaten. Fortschr Röntgenstr 2018. doi:10.1055/ s-0043-118127

[5] Schäfer SB, Rudolph C, Kolodziej M et al. Optimierung von GanzkörperCT-Untersuchungen an Polytraumatisierten anhand des Vergleichs mit den aktuellen diagnostischen Referenzwerten. Fortschr Röntgenstr 2019. doi:10.1055/a-0881-3113

[6] George A], Manghat NE, Hamilton MCK. Comparison between a fixeddose contrast protocol and a weight-based contrast dosing protocol in abdominal CT. Clin Radiol 2016. doi:10.1016/j.crad.2016.07.009

[7] Benbow M, Bull RK. Simple weight-based contrast dosing for standardization of portal phase CT liver enhancement. Clin Radiol 2011. doi:10.1016/j.crad.2010.12.022

[8] Feng ST, Zhu H, Peng Z et al. An Individually Optimized Protocol of Contrast Medium Injection in Enhanced CT Scan for Liver Imaging. Contrast Media Mol Imaging 2017. doi:10.1155/2017/7350429

[9] Svensson A, Thor D, Fischer MA et al. Dual source abdominal computed tomography: the effect of reduced $\mathrm{X}$-ray tube voltage and intravenous contrast media dosage in patients with reduced renal function. Acta Radiol 2019. doi:10.1177/0284185118783213

[10] Megibow Alec J, Jacob G, Heiken JP. Quantitative and Qualitative Evaluation of Volume of Low Osmolality Contrast Medium Needed for Routine Helical Abdominal CT. Am J Roentgenol 2001. doi:10.2214/ajr.176.3.1760583

[11] Perrin E, Jackson M, Grant R et al. Weight-adapted iodinated contrast media administration in abdomino-pelvic CT: Can image quality be maintained? Radiography (Lond) 2018. doi:10.1016/j.radi.2017.08.011

[12] Pfitzner C, May S, Nüchter A. Body Weight Estimation for Dose-Finding and Health Monitoring of Lying, Standing and Walking Patients Based on RGB-D Data. Sensors (Basel, Switzerland) 2018. doi:10.3390/s18051311

[13] Saltybaeva N, Schmidt B, Wimmer A et al. Precise and Automatic Patient Positioning in Computed Tomography: Avatar Modeling of the Patient
Surface Using a 3-Dimensional Camera. Invest Radiol 2018. doi:10.1097/RLI.0000000000000482

[14] Toth T, Ge Z, Daly MP. The influence of patient centering on CT dose and image noise. Med Phys 2007. doi:10.1118/1.2748113

[15] Statistisches Bundesamt. Körpermaße nach Altersgruppen und Geschlecht. https://www.destatis.de/DE/Themen/Gesellschaft-Umwelt/ Gesundheit/Gesundheitszustand-Relevantes-Verhalten/Tabellen/ liste-koerpermasse.html

[16] Descoteaux M, Maier-Hein L, Franz A et al. Medical Image Computing and Computer-Assisted Intervention - MICCAI 2017. Cham: Springer International Publishing; 2017. doi:10.1007/978-3-319-66185-8

[17] Kondo H, Kanematsu M, Goshima S et al. Abdominal multidetector CT in patients with varying body fat percentages: estimation of optimal contrast material dose. Radiology 2008. doi:10.1148/radiol.2492080033

[18] Fleischmann D, Kamaya A. Optimal vascular and parenchymal contrast enhancement: the current state of the art. Radiol Clin North Am 2009. doi:10.1016/j.rcl.2008.10.009

[19] Boland GWL, Houghton MP, Marchione DG et al. Maximizing outpatient computed tomography productivity using multiple technologists. Journal of the American College of Radiology: JACR 2008. doi:10.1016/ j.jacr.2007.07.009

[20] Bégin A, Martel G, Lapointe R et al. Accuracy of preoperative automatic measurement of the liver volume by $\mathrm{CT}$-scan combined to a 3D virtual surgical planning software (3DVSP). Surg endosc 2014. doi:10.1007/ s00464-014-3611-x

[21] Vauthey JN, Chaoui A, Do KA et al. Standardized measurement of the future liver remnant prior to extended liver resection: methodology and clinical associations; 2000. doi:10.1067/msy.2000.105294

[22] Hoffmann RT, Jakobs TF, Tatsch $\mathrm{K}$ et al. Selektive interne Radiotherapie bei fortgeschrittenen Lebertumoren und Metastasen. Dtsch Med Wochenschr 2008. doi:10.1055/s-0028-1091244

[23] Urata K, Kawasaki S, Matsunami $\mathrm{H}$ et al. Calculation of child and adult standard liver volume for liver transplantation. Hepatology 1995. doi:10.1002/hep.1840210515

[24] Bundesamt für Strahlenschutz. Leitfaden zur Handhabung der diagnostischen Referenzwerte in der Röntgendiagnostik. https://www.bfs.de/ SharedDocs/Downloads/BfS/DE/fachinfo/ion/leitfaden-drw-roe.pdf? _blob=publicationFile\&v $=11$ 